\title{
VAGO Method for the Solution of Elliptic Second-Order Boundary Value Problems
}

\author{
N. Vabishchevich ${ }^{1}$ and P. Vabishchevich ${ }^{2}$ \\ ${ }^{1}$ Nuclear Safety Institute \\ 52, B. Tulskaya, 115191 Moscow, Russia \\ E-mail: nvab@ibrae.ac.ru \\ ${ }^{2}$ Keldysh Institute of Applied Mathematics \\ 4-A Miusskaya Square, 125047 Moscow, Russia \\ E-mail(corresp.): vabishchevich@gmail.com
}

Received July 23, 2010; revised September 20, 2010; published online November 15, 2010

\begin{abstract}
Mathematical physics problems are often formulated using differential operators of vector analysis, i.e. invariant operators of first order, namely, divergence, gradient and rotor (curl) operators. In approximation of such problems it is natural to employ similar operator formulations for grid problems. The VAGO (Vector Analysis Grid Operators) method is based on such a methodology. In this paper the vector analysis difference operators are constructed using the Delaunay triangulation and the Voronoi diagrams. Further the VAGO method is used to solve approximately boundary value problems for the general elliptic equation of second order. In the convection-diffusion-reaction equation the diffusion coefficient is a symmetric tensor of second order.
\end{abstract}

Keywords: finite difference method, unstructured grids, Delaunay triangulation, Voronoi diagrams, convection-diffusion problems.

AMS Subject Classification: 65N06; 65M06.

\section{Introduction}

In the theory of difference schemes [19] the problem of approximation of a differential problem via discrete problem received primary emphasis. A general method of balance (integro-interpolation method) is used as the basic one to construct discrete analogs. This method was proposed by Samarskii [18] and currently it is known as the finite volume method [4, 10, 11]. A difference scheme is constructed using the balance method via integration of the initial equation over a control volume - a part of the computational domain adjacent to the specified grid point.

In solving mathematical physics problems on general unstructured grids, 
the Delaunay triangulation is often used. It displays some optimal features [6]. For the Delaunay triangulation, it is natural to consider as control volumes a Voronoi polygon - a set of points laying closer to the considered grid point comparing with all other grid points. Consistent approximations of the main vector operators on the basis of the Delaunay triangulation were constructed in [21]. A systematic investigation of approximations for gradient and divergence operators on dual Voronoi-Delaunay partitionings has been conducted in [14, 15] for model $2 \mathrm{D}$ and $3 \mathrm{D}$ problems.

The present work continues our studies on Vector Analysis Grid Operators method. In paper [22], we have discussed problems of constructing consistent approximations for basic operators of vector analysis, i.e. gradient, divergence and rotor operators. This direction of research most closely related to the mimetic discretization methods using the difference approximations [17] and finite element exterior calculus in finite element approximations [1]. Special representations were derived for truncation errors that allowed us to obtain the corresponding estimates for the convergence rate of the approximate solution to the exact one.

Possibilities of the VAGO method have been demonstrated on some scalar and vector problems. Difference schemes for steady-state convection-diffusion problems were constructed on the basis of the dual Voronoi-Delaunay partitioning in $[13,23]$. In these problems (see, e.g., [9]) the emphasis is on properties of discrete operators for the convection and diffusion transport. The convective terms are written $[20,23]$ in the divergent (conservative), non-divergent (characteristic) and symmetric forms. In [22], the VAGO type approximations were designed and truncation errors were investigated for the Dirichlet problem for stationary and unsteady convection-diffusion problems for isotropic media.

Particular emphasis should be placed on approximation of diffusion transport operator for an anisotropic medium where the diffusion coefficient is a symmetric tensor of second order. In this case we should control at the discrete level not only the self-adjoint property of the grid diffusion operator but the monotonicity and conservation properties for the corresponding grid approximations, too [19]. The state of art in approximation of problems with mixed derivatives on rectangular grids is given in [12]. Properties of standard finite-element approximations on triangular or tetrahedral meshes are discussed in detail in [16]. To obtain the monotonicity property, a modification of standard linear Galerkin finite element discretizations of the Laplace operator was performed. Much attention is given to constructing finite volume schemes for the approximate solution of convection-diffusion boundary value problems with an anisotropic diffusion coefficient (see [3] and references therein). In particular (see, $[5]$ ), the finite volume schemes are developed with an anisotropic diffusion for approximation of local discrete gradients. The results of work [14] are generalized in [8] to the $2 \mathrm{D}$ div-curl system in anisotropic media. In constructing the finite element schemes (see, for example, [16]) and the finite volume schemes (see $[4,5])$ for problems with anisotropic diffusion the variational formulation of the given problem is commonly used. This allows us to consider only the gradient approximation only. In VAGO method we build not only the gradient, but the divergence operator with the explicit form of truncation error. 
The outline of this paper is the following. In Section 2, the boundaryvalue problem for the general elliptic equation of second order is formulated in an invariant form as a problem for a convection-diffusion equation based on vector analysis operators divergence and gradient. The diffusion coefficient is a symmetric positive definite tensor of second order. In Section 3, on dual Voronoi-Delaunay partitionings the corresponding spaces of grid functions are introduced. Using the VAGO method the difference operators of divergence and gradient are constructed and investigated in Section 4. Difference schemes for the considered convection-diffusion-reaction problem are studied in Section 5. Approximation of the diffusion transport operator with an anisotropic diffusion coefficient has received much consideration.

\section{The Boundary Value Problem}

The boundary value problem is considered for an elliptic equation of second order. In $n$-dimensional bounded domain $\Omega$ with smooth enough boundary $\partial \Omega$ function $u(\mathbf{x}), \mathbf{x}=\left(x_{1}, \ldots, x_{n}\right)$ satisfies the following equation

$$
\begin{array}{r}
-\sum_{\alpha, \beta=1}^{n} \frac{\partial}{\partial x_{\alpha}}\left(d_{\alpha \beta}(\mathbf{x}) \frac{\partial u}{\partial x_{\beta}}\right)+\sum_{\alpha=1}^{n} \frac{\partial}{\partial x_{\alpha}}\left(a_{\alpha} u\right)+\sum_{\alpha=1}^{n} b_{\alpha} \frac{\partial u}{\partial x_{\alpha}}+c(\mathbf{x}) u(\mathbf{x})=f(\mathbf{x}), \\
\mathbf{x} \in \Omega .
\end{array}
$$

The following ellipticity and symmetry conditions for coefficients at the higher order derivatives and positivity condition for convection-reaction coefficients are satisfied:

$$
\begin{aligned}
& d_{\alpha \beta}=d_{\beta \alpha}, \quad \sum_{\alpha, \beta=1}^{n} d_{\alpha \beta}(\mathbf{x}) y_{\alpha} y_{\beta} \geq \delta \sum_{\alpha=1}^{n} y_{\alpha}^{2}, \quad \delta>0, \quad \forall \mathbf{y} \in \mathbb{R}^{n}, \quad \forall \mathbf{x} \in \bar{\Omega}, \\
& \frac{1}{2} \sum_{\alpha=1}^{n} \frac{\partial\left(a_{\alpha}-b_{\alpha}\right)}{\partial x_{\alpha}}+c(\mathbf{x}) \geq 0 .
\end{aligned}
$$

Assume that the right hand side of the equation can be represented as follows

$$
f(\mathbf{x})=f_{0}(\mathbf{x})+\sum_{\alpha=1}^{n} \frac{\partial f_{\alpha}}{\partial x_{\alpha}}
$$

The simplest boundary value problem is considered for (2.1) with the following boundary conditions

$$
u(\mathbf{x})=0, \quad \mathbf{x} \in \partial \Omega .
$$

In Hilbert space $L_{2}(\Omega)$ the scalar product and norm are introduced by

$$
(u, v)=\int_{\Omega} u(\mathbf{x}) v(\mathbf{x}) d \mathbf{x}, \quad\|u\|=(u, u)^{1 / 2} .
$$

In $\mathcal{H}$ (grad) we introduce

$$
(u, v)_{\mathcal{H}(\text { grad })}=(u, v)+\sum_{\alpha=1}^{n}\left(\frac{\partial u}{\partial x_{\alpha}}, \frac{\partial u}{\partial x_{\alpha}}\right), \quad\|u\|_{\mathcal{H}(\text { grad })}=\left((u, u)_{\mathcal{H}(\text { grad })}\right)^{1 / 2} .
$$


For the solution of problem (2.1)-(2.3) the following apriori estimate takes place (see, e.g., [7])

$$
\|u\|_{\mathcal{H}(\mathrm{grad})} \leq M\left(\sum_{\alpha=0}^{n}\left\|f_{\alpha}\right\|^{2}\right)^{1 / 2} .
$$

Unstructured computational grids are often used to solve approximately boundary problems of mathematical physics. In some cases it is not suitable to use the coordinate-wise form of the considering equations and boundary conditions in one or another coordinate system. It seems more reasonable to employ the invariant formulation of the problem. In this case the considered problem is represented via operators of vector and tensor analysis. Equation (2.1) is the basic one for problems of continuum mechanics. Vectors

$$
\mathbf{a}=\left\{a_{\alpha}\right\}, \quad \mathbf{b}=\left\{b_{\alpha}\right\}, \quad \alpha=1, \ldots, n,
$$

are connected with the convective transport. Symmetric tensor of second rank

$$
\mathbf{D}=\left\{d_{\alpha \beta}\right\}, \quad \alpha=1, \ldots, n, \quad \beta=1, \ldots, n
$$

in (2.1) corresponds to the diffusion transport. Coefficient $c(\mathbf{x})$ can be treated as a reaction. In such an interpretation equation (2.1) is nothing but the equation of convection-diffusion-reaction

$$
-\operatorname{div}(\mathbf{D} \operatorname{grad} u)+\operatorname{div}(\mathbf{a} u)+\mathbf{b} \operatorname{grad} u+c(\mathbf{x}) u(\mathbf{x})=f(\mathbf{x}), \quad \mathbf{x} \in \Omega .
$$

Boundary value problem (2.1)-(2.3) in form (2.3), (2.4) does not employ explicitly Cartesian coordinates $x_{1}, \ldots, x_{n}$.

\section{Delaunay Triangulation and Voronoi Diagrams}

Assume that the computational domain is a convex polyhedron $\Omega, n=2,3$ with boundary $\partial \Omega$. In domain $\bar{\Omega}=\Omega \cup \partial \Omega$ we consider the grid $\bar{\omega}$, which consists of nodes $\mathbf{x}_{i}^{D}, i=1,2, \ldots, M_{D}$, and the angles of the polyhedron $\Omega$ are nodes. Let $\omega$ be a set of inner nodes and $\partial \omega$ is a set of boundary nodes, i.e., $\omega=\bar{\omega} \cap \Omega, \partial \omega=\bar{\omega} \cap \partial \Omega$.

Each node $\mathbf{x}_{i}^{D}, i=1,2, \ldots, M_{D}$, connects a certain part of the computational domain, namely, the Voronoi polyhedron or its part belonging to $\Omega$. The Voronoi polyhedron (polygon) for a separate node is a set of points laying closer to this node than to all other ones:

$V_{i}=\left\{\mathbf{x}|\mathbf{x} \in \Omega,| \mathbf{x}-\mathbf{x}_{i}^{D}|<| \mathbf{x}-\mathbf{x}_{j}^{D} \mid, j=1,2, \ldots, M_{D}\right\}, \quad i=1,2, \ldots, M_{D}$,

where $|\cdot|$ is the Euclidean distance. Each vertex $\mathbf{x}_{k}^{V}, k=1,2, \ldots, M_{V}$ of the Voronoi polyhedron is associated with the tetrahedron constructed by the appropriate nodes contacting the Voronoi polyhedrons. We will assume that all vertices of the Voronoi polyhedrons lay either inside the computational domain $\Omega$ or on its boundary $\partial \Omega$. These tetrahedrons determine the Delaunay triangulation - a dual triangulation to the Voronoi diagram. The $D$-grid in the domain $\Omega$ is determined by the set of nodes (vertices of tetrahedrons of the 
Delaunay triangulation) $\mathbf{x}_{i}^{D}, i=1,2, \ldots, M_{D}$, the $V$-grid is defined by the set of nodes (vertices of polyhedron of the Voronoi diagram) $\mathbf{x}_{k}^{V}, k=1,2, \ldots, M_{V}$.

We denote by $D_{k}$ a separate tetrahedron of the Delaunay triangulation. This tetrahedron is identified by the number $k$ of the Voronoi polyhedron vertex, $k=1,2, \ldots, M_{V}$. The tetrahedrons $D_{k}, k=1,2, \ldots, M_{V}$ cover the entire computational domain:

$$
\bar{\Omega}=\cup_{k=1}^{M_{V}} \bar{D}_{k}, \bar{D}_{k}=D_{k} \cup \partial D_{k}, D_{k} \cap D_{m}=\emptyset, k \neq m, k, m=1,2, \ldots, M_{V} .
$$

For common planes of the tetrahedron we use the notations

$$
\partial D_{k m}=\partial D_{k} \cap \partial D_{m}, \quad k \neq m, \quad k, m=1,2, \ldots, M_{V} .
$$

The boundary of the computational domain $\partial \Omega$ consists of the planes of Delaunay tetrahedrons. Let

$$
\partial D_{0}=\partial \Omega, \quad \partial D_{k 0}=\partial D_{k} \cap \partial D_{0}, \quad k=1,2, \ldots, M_{V} .
$$

We associate the Voronoi polyhedron $V_{i}, i=1,2, \ldots, M_{D}$ with the node of the main grid $i$. Thus, we have

$$
\begin{aligned}
& \bar{\Omega}=\bigcup_{i=1}^{M_{D}} \bar{V}_{i}, \quad \bar{V}_{i}=V_{i} \cup \partial V_{i}, \quad V_{i} \cap V_{j}=\emptyset, \quad i \neq j, \quad i, j=1,2, \ldots, M_{D}, \\
& \partial V_{i j}=\partial V_{i} \cap \partial V_{j}, \quad i \neq j, \quad i, j=1,2, \ldots, M_{D} .
\end{aligned}
$$

For each tetrahedron $D_{k}, k=1,2, \ldots, M_{V}$, we define the set of neighbours $\mathcal{W}^{D}(k)$, having common planes with $D_{k}$, i.e.,

$$
\mathcal{W}^{D}(k)=\left\{m \mid \partial D_{k} \cap \partial D_{m} \neq \emptyset, m=0,1, \ldots, M_{V}\right\}, \quad k=1,2, \ldots, M_{V} .
$$

In this case, $m=0$ means that the tetrahedron $D_{k}$ contacts the boundary. We define also the neighbours for each Voronoi polyhedron $V_{i}, i=1,2, \ldots, M_{D}$ :

$$
\mathcal{W}^{V}(i)=\left\{j \mid \partial V_{i} \cap \partial V_{j} \neq \emptyset, j=1,2, \ldots, M_{D}\right\}, \quad i=1,2, \ldots, M_{D} .
$$

We assume that the introduced Delaunay triangulation and the Voronoi diagram are regular [2]. Let $h_{k}^{D}=\operatorname{diam}\left(D_{k}\right)$ denotes the diameter of $D_{k}$,

$$
\varrho_{k}^{D}=\sup \left\{\operatorname{diam}(S) \mid S-\text { sphere in } D_{k}\right\}, \quad k=1,2, \ldots, M_{V},
$$

then the regularity condition of the Delaunay triangulation is given by

$$
h_{k}^{D} / \varrho_{k}^{D} \leq \sigma>0, \quad k=1,2, \ldots, M_{V}
$$

Likewise, for the Voronoi diagram we denote by $h_{i}^{V}=\operatorname{diam}\left(V_{i}\right)$ the diameter of $V_{i}$, then

$$
\begin{aligned}
& \varrho_{i}^{V}=\sup \left\{\operatorname{diam}(S) \mid S-\text { sphere in } V_{i}\right\}, \\
& h_{i}^{V} / \varrho_{i}^{V} \leq \sigma>0, \quad i=1,2, \ldots, M_{D},
\end{aligned}
$$


and $h=\max _{i, k}\left\{h_{i}^{V}, h_{k}^{D}\right\}$,

$$
\operatorname{meas}\left(D_{k}\right)=\int_{D_{k}} d \mathbf{x}, \quad k=1,2, \ldots, M_{V}, \quad \operatorname{meas}\left(V_{i}\right)=\int_{V_{i}} d \mathbf{x}, \quad i=1,2, \ldots, M_{D} .
$$

We will approximate the scalar functions of the continuous argument by the scalar grid functions that are defined in the nodes of the $D$-grid or in the nodes of the $V$-grid. We denote by $H_{D}$ the set of grid functions defined on the $D$-grid

$$
H_{D}=\left\{y(\mathbf{x}) \mid y(\mathbf{x})=y\left(\mathbf{x}_{i}^{D}\right)=y_{i}^{D}, i=1,2, \ldots, M_{D}\right\} .
$$

For the functions $y(\mathbf{x}) \in H_{D}$, vanishing on the boundary $\partial \omega$, we define

$$
H_{D}^{0}=\left\{y(\mathbf{x}) \mid y(\mathbf{x}) \in H_{D}, y(\mathbf{x})=0, \mathbf{x} \in \partial \omega\right\} .
$$

We consider the scalar product and the norm for the scalar grid functions from $H_{D}$ by

$$
(y, v)_{D}=\sum_{i=1}^{M_{D}} y_{i}^{D} v_{i}^{D} \operatorname{meas}\left(V_{i}\right), \quad\|y\|_{D}=(y, y)_{D}^{1 / 2}
$$

This scalar product and the norm are grid analogs of the scalar product and the $L_{2}(\Omega)$-norm for the scalar functions of the continuous argument.

To determine the vector field in the control volume, it is natural to use the components of the sought function normal to the corresponding planes of the control volume. Choosing the initial and the final node, we connect with each tetrahedron edge $D_{k}, k=1,2, \ldots, M_{V}$ or polyhedron edge $V_{i}, i=1,2, \ldots, M_{D}$. For Delaunay triangulation the normals to the planes are the directed edges of Voronoi diagram and vice versa. For the approximation of the vector functions thereby we can use projections of the vectors on the directed edges. We will further use exactly this variant with the description of the vector field in the control volume by means of vector projections on the edges of the control volume.

We will orient the Delaunay triangulation edges by the unit vector

$$
\mathbf{e}_{i j}^{D}=\mathbf{e}_{j i}^{D}, \quad i=1,2, \ldots, M_{D}, \quad j \in \mathcal{W}^{V}(i),
$$

directed from the node with a smaller index number to the node with a larger index. The vector function $\mathbf{y}(\mathbf{x})$ on the Delaunay triangulation is defined by the components $y_{i j}^{D}=\mathbf{y} \mathbf{e}_{i j}^{D}, i=1,2, \ldots, M_{D}, j \in \mathcal{W}^{V}(i)$, that are given in the middle of the edges $\mathbf{x}_{i j}^{D}=\frac{1}{2}\left(\mathbf{x}_{i}^{D}+\mathbf{x}_{j}^{D}\right)$. For the Delaunay triangulation and the Voronoi diagram we define the length of the edges in the following way:

$$
l_{i j}^{D}=\left|\mathbf{x}_{i}^{D}-\mathbf{x}_{j}^{D}\right|, \quad i=1,2, \ldots, M_{D}, \quad j \in \mathcal{W}^{V}(i) .
$$

We denote by $\mathbf{H}_{D}$ the set of grid vector functions determined by the components $y_{i j}^{D}, i=1,2, \ldots, M_{D}, j \in \mathcal{W}^{V}(i)$ that are given in the middle of the edges. In a similar way we denote by $\mathbf{H}_{V}$ the set of grid vector functions defined by the components $y_{k m}^{V}, k=1,2, \ldots, M_{V}, m \in \mathcal{W}^{D}(k)$. If the tangential 
components of the grid vector functions $\mathbf{y} \in \mathbf{H}_{D}$ vanish on the boundary, we define

$\mathbf{H}_{D}^{0}=\left\{\mathbf{y} \mid \mathbf{y} \in \mathbf{H}_{D}, \mathbf{y}(\mathbf{x}) \mathbf{e}_{i j}^{D}=0, \mathbf{x}=\mathbf{x}_{i j}^{D} \in \partial \omega, i=1,2, \ldots, M_{D}, j \in \mathcal{W}^{V}(i)\right\}$.

Consider the scalar product and norm in $\mathbf{H}_{D}$ :

$$
(\mathbf{y}, \mathbf{v})_{D}=\sum_{i=1}^{M_{D}} \sum_{j \in \mathcal{W}^{V}(i)} y_{i j}^{D} v_{i j}^{D}\left|\mathbf{x}_{i}^{D}-\mathbf{x}_{i j}^{D}\right| \operatorname{meas}\left(\partial V_{i j}\right), \quad\|\mathbf{y}\|_{D}=(\mathbf{y}, \mathbf{y})_{D}^{1 / 2}
$$

\section{Gradient and Divergence Approximations}

Let us present the main results (see [22] for details) connected with approximation of gradient and divergence operators on the basis of the Delaunay triangulation and Voronoi diagram.

The set of grid functions $H_{D}$ can be the domain of definition of the grid gradient operator. We denote the grid gradient operator by $\operatorname{grad}_{D}$. Taking into account the chosen edge orientation, at the points $\mathbf{x}_{i j}^{D}$ we set

$$
\left(\operatorname{grad}_{D} y\right)_{i j}^{D}=\eta(i, j) \frac{y_{j}^{D}-y_{i}^{D}}{l_{i j}}, \quad i=1,2, \ldots, M_{D}, \quad j \in \mathcal{W}^{V}(i),
$$

i.e., the range of values of the operator $\operatorname{grad}_{D}: H_{D} \rightarrow \mathbf{H}_{D}$ is the set of vector grid functions $\mathbf{H}_{D}$. In (4.1), we use the following notation:

$$
\eta(i, j)=\left\{\begin{array}{rr}
1, & j>i \\
-1, & j<i
\end{array}\right.
$$

For the truncation error of the grid operator $\operatorname{grad}_{D}$ we have

$$
\begin{gathered}
\left(\operatorname{grad}_{D} u\right)(\mathbf{x})=(\operatorname{grad} u)(\mathbf{x})+\mathbf{g}(\mathbf{x}), \quad \mathbf{g}=\mathcal{O}\left(h^{2}\right), \quad \mathbf{x}=\mathbf{x}_{i j}^{D} \\
i=1,2, \ldots, M_{D}, \quad j \in \mathcal{W}^{V}(i)
\end{gathered}
$$

provided that $u(\mathbf{x})$ is a sufficiently smooth functions.

For the Voronoi polyhedron this equality is written in the following form:

$$
\int_{V_{i}} \operatorname{div} \mathbf{u} d \mathbf{x}=\sum_{j \in \mathcal{W}^{V}(i)} \int_{\partial V_{i j}}\left(\mathbf{u} \cdot \mathbf{n}_{i j}^{V}\right) d \mathbf{x},
$$

where $\mathbf{n}_{i j}^{V}$ is the normal to the edge $\partial V_{i j}$ outside with respect to $V_{i}$. To construct the grid operator $\operatorname{div}_{D}: \mathbf{H}_{D} \rightarrow H_{D}$ we use the elementary formulas of integration for the left- and right-hand sides (4.2). This leads to the grid analogs of the operator div in the form of

$$
\left(\operatorname{div}_{D} \mathbf{y}\right)_{i}^{D}=\frac{1}{\operatorname{meas}\left(V_{i}\right)} \sum_{j \in \mathcal{W}^{V}(i)}\left(\mathbf{n}_{i j}^{V} \cdot \mathbf{e}_{i j}^{D}\right) y_{i j}^{D} \operatorname{meas}\left(\partial V_{i j}\right), \quad i=1,2, \ldots, M_{D}
$$


We have

$$
\begin{aligned}
& \left(\operatorname{div}_{D} \mathbf{u}\right)(\mathbf{x})=(\operatorname{div} \mathbf{u})(\mathbf{x})+g(\mathbf{x})+\left(\operatorname{div}_{D} \mathbf{q}\right)(\mathbf{x}), \\
& g=\mathcal{O}(h), \quad \mathbf{q}=\mathcal{O}(h), \quad \mathbf{x}=\mathbf{x}_{i}^{D}, \quad i=1,2, \ldots, M_{D}
\end{aligned}
$$

for the truncation error. The truncation error for the grid divergence operator $\operatorname{div}_{D}$ equals $\mathcal{O}(1)$. However, there exists a special divergence expression of the truncation error saving the situation in the case of approximation of problems of mathematical physics.

Let us highlight the adjoint property of the considered grid operators of gradient and divergence. Taking into account the above notations we have

$$
\begin{aligned}
& \left(v, \operatorname{div}_{D} \mathbf{y}\right)_{D}+\left(\operatorname{grad}_{D} v, \mathbf{y}\right)_{D}=\sum_{i \in \partial \omega} \\
& \times \sum_{j \in \mathcal{W}^{V}(i), j \in \partial \omega}\left(\mathbf{n}_{i j}^{V} \cdot \mathbf{e}_{i j}^{D}\right) \frac{v_{j}^{D}+v_{i}^{D}}{2} y_{i j}^{D} \operatorname{meas}\left(\partial V_{i j}\right) .
\end{aligned}
$$

From (4.5) it follows that $\operatorname{div}_{D}^{*}=-\operatorname{grad}_{D}$ on the set of grid functions $v \in H_{D}^{0}$ and $\mathbf{y} \in \mathbf{H}_{D}$.

\section{Approximation of the Convection-Diffusion-Reaction Problem}

In domain $\Omega$ we consider the regular $D$-grid and $V$-grid. Taking into account boundary condition (2.3), we find the approximate solution of problem (2.3), (2.4) as grid function $y(\mathbf{x}) \in H_{D}^{0}$.

A particular discussion should be given to approximation of convective, and moreover, diffusive terms in equation (2.4). We consider approximations of the convective transport operator both in the divergent and in non-divergent (characteristic) form:

$$
\mathcal{C}_{1}(\mathbf{v}) u=\mathbf{v} \operatorname{grad} u, \quad \mathcal{C}_{2}(\mathbf{v}) u=\operatorname{div}(\mathbf{v} u)
$$

at specified vector field $\mathbf{v}(\mathbf{x}), \mathbf{x} \in \Omega$.

The tangential components are approximated at the edge midpoints of the Delaunay triangulation as follows

$$
a_{i j}^{D}=\frac{1}{\operatorname{meas}\left(\partial V_{i j}\right)} \int_{\partial V_{i j}} \mathbf{a}(\mathbf{x}) \mathbf{e}_{i j}^{D} d \mathbf{x}, \quad i=1,2, \ldots, M_{D}, \quad j \in \mathcal{W}^{V}(i)
$$

For scalar functions it is natural to introduce $y\left(\mathbf{x}_{i j}^{D}\right)=\frac{1}{2}\left(y\left(\mathbf{x}_{i}^{D}\right)+y\left(\mathbf{x}_{j}^{D}\right)\right)$. The direct approximation of the convective transport operator in the divergent form results in

$$
\begin{aligned}
\mathcal{C}_{2}^{D}\left(\mathbf{v}^{D}\right) y & =\operatorname{div}_{D}\left(\mathbf{v}^{D} y\right) \\
& =\frac{1}{2 \operatorname{meas}\left(V_{i}\right)} \sum_{j \in \mathcal{W}^{V}(i)}\left(\mathbf{n}_{i j}^{V} \cdot \mathbf{e}_{i j}^{D}\right) v_{i j}^{D}\left(y_{j}^{D}+y_{i}^{D}\right) \operatorname{meas}\left(\partial V_{i j}\right) .
\end{aligned}
$$


As for the convective transport operator in the non-divergent form, its approximation is performed [23] using the grid analog of the following equality

$$
\mathcal{C}_{1}(\mathbf{v}) u=\mathcal{C}_{2}(\mathbf{v}) u-u \operatorname{div} \mathbf{v} .
$$

At $\mathcal{C}_{1}^{D}\left(\mathbf{v}^{D}\right) y=\mathcal{C}_{2}^{D}\left(\mathbf{v}^{D}\right) y-y \operatorname{div}_{D} \mathbf{v}^{D}$ we have

$$
\left(\mathcal{C}_{1}^{D}\left(\mathbf{v}^{D}\right) \mathbf{y}\right)_{i}^{D}=\frac{1}{2 \operatorname{meas}\left(V_{i}\right)} \sum_{j \in \mathcal{W}^{V}(i)}\left(\mathbf{n}_{i j}^{V} \cdot \mathbf{e}_{i j}^{D}\right) v_{i j}^{D}\left(y_{j}^{D}-y_{i}^{D}\right) \operatorname{meas}\left(\partial V_{i j}\right)
$$

For the truncation error similarly to (4.4) we have

$$
\begin{aligned}
& \mathcal{C}_{\alpha}^{D}\left(\mathbf{v}^{D}\right) u=\mathcal{C}_{\alpha}(\mathbf{v}) u+g(\mathbf{x})+\left(\operatorname{div}_{D} \mathbf{q}\right)(\mathbf{x}), \quad \alpha=1,2, \\
& g=\mathcal{O}(h), \quad \mathbf{q}=\mathcal{O}(h), \quad \mathbf{x}=\mathbf{x}_{i}^{D}, \quad i=1,2, \ldots, M_{D} .
\end{aligned}
$$

Let us construct a grid analog of the diffusion transport operator

$$
\mathcal{D} u=\operatorname{div}(\mathbf{D} \operatorname{grad} u), \quad \mathbf{x} \in \Omega .
$$

We put formally

$$
\mathcal{D}^{D} y=\operatorname{div}_{D}\left(\mathbf{D}^{D} \operatorname{grad}_{D} y\right) .
$$

Above (see also (4.1)) we have presented the grid approximation for the projection of the gradient operator onto edges of the Delaunay triangulation. Such an approximation is sufficient for approximation of the diffusion operator in isotropic media with a scalar diffusion coefficient. For general elliptic equations with mixed derivatives (2.1) with a tensor diffusion coefficient it is necessary to approximate the gradient not only along the edges of triangulation. To investigate this problem, let us start from the $2 \mathrm{D}$ case when $n=2$, see Fig. 1 .

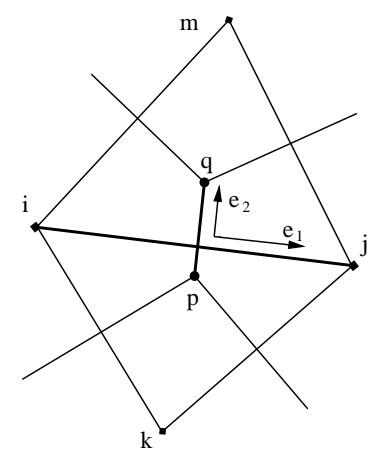

Figure 1. Two-dimensional case.

Here $i, j, k, m$ are nodes of the Delaunay triangulation, $i, j, k$ and $i, j, m$ are two adjacent triangles of this triangulation with common edge $(i, j)$, and $p$, $q$ denote nodes of the Voronoi diagram. For nodes of the Voronoi diagram, 
notations $\mathbf{x}_{p}^{V}=\mathbf{x}_{i j k}^{D}$ and $\mathbf{x}_{q}^{V}=\mathbf{x}_{i j m}^{D}$ are used. Highlighted in Fig. 1 edges of the Delaunay triangulation and Voronoi diagram are orthogonal one to another. At the point of their intersection (i.e., at point $\mathbf{x}=\mathbf{x}_{i j}^{D}$ ) we introduce a local coordinate system $\left(s_{1}, s_{2}\right)$ with direction unit vectors $\left.\mathbf{e}_{1}, \mathbf{e}_{2}\right)$.

Divergence of a vector field at the discrete level (see (4.3)) is defined using the tangential component. To approximate the diffusion transport, it is necessary to evaluate the diffusion tensor (5.3). For the introduced local coordinate system we take

$$
(\mathbf{D})_{i j}^{D}=\left(\begin{array}{ll}
\left(d_{11}^{D}\right)_{i j} & \left(d_{12}^{D}\right)_{i j} \\
\left(d_{21}^{D}\right)_{i j} & \left(d_{22}^{D}\right)_{i j}
\end{array}\right) .
$$

Coefficients $\left(d_{\alpha \beta}^{D}\right)_{i j}, \alpha, \beta=1,2$ are calculated via specified smooth enough coefficients $d_{\alpha \beta}(\mathbf{x}), \alpha, \beta=1,2$ of equation (2.1). If the local coordinate system $\left(s_{1}, s_{2}\right)$ coincides with $\left(x_{1}, x_{2}\right)$, then we have that $\left(d_{\alpha \beta}^{D}\right)_{i j}=d_{\alpha \beta}\left(\mathbf{x}_{i j}^{D}\right), \alpha, \beta=$ 1,2 . In a general case the recalculation rule for a tensor of the second order should be taken into account when we use a new orthogonal coordinate system. The tangential component of the flow can be written as

$$
\left(\mathbf{D} \operatorname{grad}_{D} y\right)_{i j}^{D}=\left(d_{11}^{D}\right)_{i j}\left(\operatorname{grad}_{D} y\right)_{1}+\left(d_{12}^{D}\right)_{i j}\left(\operatorname{grad}_{D} y\right)_{2} .
$$

The tangential component of the gradient is given by

$$
\left(\operatorname{grad}_{D} y\right)_{1}=\left(\operatorname{grad}_{D} y\right)_{i j}^{D}=\frac{y_{j}^{D}-y_{i}^{D}}{l_{i j}^{D}} .
$$

For the normal component we have

$$
\left(\operatorname{grad}_{D} y\right)_{2}=\frac{y_{q}^{V}-y_{p}^{V}}{l_{p q}^{V}},
$$

where $l_{p q}^{V}=\left|\mathbf{x}_{i j m}^{D}-\mathbf{x}_{i j k}^{D}\right|$ is the edge length of the Voronoi polygon.

Evaluation of the normal component of the gradient via (5.6) is based on interpolation of values of a scalar function defined at the Delaunay triangulation nodes to the Voronoi polygon nodes. Let $l_{p i j}=\left|\mathbf{x}_{p}^{V}-\mathbf{x}_{i j}^{D}\right|$ be the distance from node $p$ of the Voronoi partitioning up to edge $(i, j)$ of the Delaunay triangulation so that $l_{p q}^{V}=d_{p i j}+d_{q i j}$. The linear interpolation with accuracy $\mathcal{O}\left(h^{2}\right)$ over triangle $D_{p}$ results in

$$
y_{p}^{V}=\frac{1}{\operatorname{meas}\left(D_{p}\right)}\left(y_{k}^{D} l_{i j}^{D} d_{p i j}+y_{i}^{D} l_{j k}^{D} d_{p j k}+y_{j}^{D} l_{i k}^{D} d_{p i k}\right) .
$$

Taking into account (5.5)-(5.7), we get from (5.4) the following representation for the truncation error of the flow:

$$
\left(\mathbf{D} \operatorname{grad}_{D} u\right)_{i j}^{D}=(\mathbf{D} \operatorname{grad} u)\left(\mathbf{x}_{i j}^{D}\right)+\mathcal{O}(h) .
$$

Approximation (2.1) leads to the grid equation

$$
\begin{array}{r}
-\operatorname{div}_{D}\left(\mathbf{D}^{D} \operatorname{grad}_{D} y\right)+\mathcal{C}_{2}^{D}\left(\mathbf{a}^{D}\right) y+\mathcal{C}_{1}^{D}\left(\mathbf{b}^{D}\right) y+c^{D} y=f^{D}, \\
\mathbf{x}=\mathbf{x}_{i}^{D}, \quad i=1,2, \ldots, M_{D},
\end{array}
$$


where, for instance, $c^{D}\left(\mathbf{x}_{i}^{D}\right)=c^{D}\left(\mathbf{x}_{i}^{D}\right)$. Grid equation (5.9) approximates equation (2.1) with error

$$
\psi(\mathbf{x})=g(\mathbf{x})+\left(\operatorname{div}_{D} \mathbf{q}\right)(\mathbf{x}), \quad g=\mathcal{O}(h), \quad \mathbf{q}=\mathcal{O}(h) .
$$

In the $3 \mathrm{D}$ case approximations of the diffusion transport operator are constructed in a similar way. Let us consider (see Fig. 2) two adjacent tetrahedrons $i, j, k, m$ and $i, j, k, n$ of the Delaunay triangulation. For the corresponding nodes of the Voronoi diagram (vortices of the Voronoi polygon) we again use notations $\mathbf{x}_{p}^{V}$ and $\mathbf{x}_{q}^{V}$. The line connecting these vortices is perpendicular to triangle $i, j, k$ and intersects it at point $r$ (see, Fig. 2). Point $s$ is the midpoint of edge $(i, j)$. Straight line $(r, s)$ is perpendicular to edge $(i, j)$ of the Delaunay triangulation and edge $(p, q)$ of the Voronoi diagram. On the basis of these three lines $(i, j),(p, q)$ and $(r, s)$ highlighted in Fig. 2 we construct a local coordinate system $\left(s_{1}, s_{2}, s_{3}\right)$ with direction unit vectors $\left.\mathbf{e}_{1}, \mathbf{e}_{2}, \mathbf{e}_{3}\right)$.

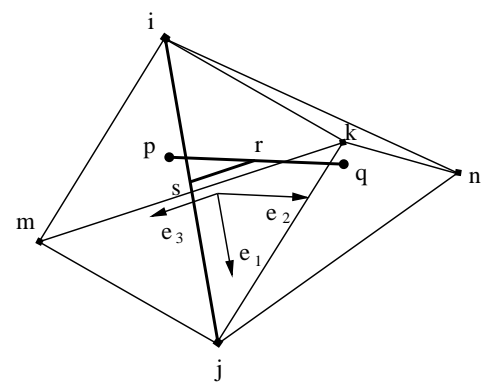

Figure 2. Tree-dimensional case.

Similarly to the $2 \mathrm{D}$ case we can evaluate now the diffusion tensor in the local coordinate system

$$
(\mathbf{D})_{i j}^{D}=\left(\begin{array}{lll}
\left(d_{11}^{D}\right)_{i j} & \left(d_{12}^{D}\right)_{i j} & \left(d_{13}^{D}\right)_{i j} \\
\left(d_{21}^{D}\right)_{i j} & \left(d_{22}^{D}\right)_{i j} & \left(d_{23}^{D}\right)_{i j} \\
\left(d_{31}^{D}\right)_{i j} & \left(d_{32}^{D}\right)_{i j} & \left(d_{33}^{D}\right)_{i j}
\end{array}\right) .
$$

For smooth enough coefficients $d_{\alpha \beta}(\mathbf{x}), \alpha, \beta=1,2,3$ of equation (2.1) and in the case of coincidence of local coordinate system $\left(s_{1}, s_{2}, s_{3}\right)$ with $\left(x_{1}, x_{2}, x_{3}\right)$ it is possible to take

$$
\left(d_{\alpha \beta}^{D}\right)_{i j}=d_{\alpha \beta}\left(\mathbf{x}_{i j}^{D}\right), \quad \alpha, \beta=1,2,3 .
$$

In local coordinate system $\left(s_{1}, s_{2}, s_{3}\right)$ the flow component along edge $(i, j)$ of the Delaunay triangulation is equal to

$$
\left(\mathbf{D} \operatorname{grad}_{D} y\right)_{i j}^{D}=\left(d_{11}^{D}\right)_{i j}\left(\operatorname{grad}_{D} y\right)_{1}+\left(d_{12}^{D}\right)_{i j}\left(\operatorname{grad}_{D} y\right)_{2}+\left(d_{13}^{D}\right)_{i j}\left(\operatorname{grad}_{D} y\right)_{3}
$$


The tangential component of gradient $\left(\operatorname{grad}_{D} y\right)_{1}$ is calculated in accordance with (5.5) whereas for component $\left(\operatorname{grad}_{D} y\right)_{2}$ (along the edge of the Voronoi diagram) approximation (5.6) is used. For the third term in (5.11) we employ

$$
\left(\operatorname{grad}_{D} y\right)_{3}=\frac{\bar{y}_{r}-\bar{y}_{s}}{l_{r s}},
$$

where $l_{r s}$ is the distance between points $r$ and $s$. Values of scalar function $\bar{y}_{r}, \bar{y}_{s}$ in (5.12) are approximated with the second order accuracy by (5.7)-type formulas using values defined at nodes of the Delaunay triangulation.

So, representation (5.8) for the truncation error of the flow is valid in the 3D case, too. Hence we also obtain grid equation (5.9) with representation (5.10) for the truncation error. On the basis of this divergent representation for the error we can prove convergence of the discrete solution to the exact one [22] in the grid analog of space $\mathcal{H}$ (grad) with the first order with respect to $h$. Let us only note that for problems with variable diffusion coefficients, we require additional analysis to check the conditions of ellipticity on the grid level.

\section{Conclusions}

To approximate the convective terms in the divergent and nondivergent forms we have constructed approximations on the Delaunay triangulation and the Voronoi diagrams. We construct a local representation with divergent terms for the approximation error.

Approximation of diffusion term with tensor diffusion coefficient for twoand three-dimensional problems using local orthogonal coordinate proposed. We prove a local error estimate for the grid fluxes.

On the basis of the proposed approximations, we construct the grid problem for the general scalar equation of diffusion-convection-reaction with explicit divergent representation for the approximation error.

\section{References}

[1] D.N. Arnold, R.S. Falk and R. Winther. Finite element exterior calculus, homological techniques, and applications. Acta Numerica, 15:1-155, 2006.

[2] P. G. Ciarlet. The Finite Element Method for Elliptic Problems. North-Holland, Amsterdam, New York, 1978.

[3] Jérôme Droniou and Robert Eymard. A mixed finite volume scheme for anisotropic diffusion problems on any grid. Numer. Math., 105(1):35-71, 2006. Doi:10.1007/s00211-006-0034-1.

[4] R. Eymard, T. Gallouët and R. Herbin. Finite volume methods. In Handbook of Numerical Analysis, volume 7, pp. 713-1020, Amsterdam, 2000. North Holland.

[5] R. Eymard, T. Gallouët and R. Herbin. A cell-centred finite-volume approximation for anisotropic diffusion operators on unstructured meshes in any space dimension. IMA J. Numer. Anal., 26(2):326-353, 2006.

[6] Paul-Louis George and Houman Borouchaki. Delaunay triangulation and meshing. Application to finite elements. Transl. from the French. Paris: Édition Hermès. vii, 413 p., 1998. 
[7] David Gilbarg and Neil S. Trudinger. Elliptic partial differential equations of second order. Grundlehren der Mathematischen Wissenschaften, 224. Berlin etc.: Springer-Verlag. XIII, 513 p., 1983.

[8] Xiaohua $\mathrm{Hu}$ and R.A. Nicolaides. Covolume techniques for anisotropic media. Numer. Math., 61(2):215-234, 1992.

[9] R. D. Lazarov and P. S. Vassilevski. Numerical methods for convection-diffusion problems on general grids. In B. Bojanov(Ed.), Approximation Theory: A volume dedicated to Blogovest Sendov, pp. 258-283, Sofia, 2002. DARBA.

[10] Randall J. Leveque. Finite volume methods for hyperbolic problems. Cambridge Texts in Applied Mathematics. Cambridge: Cambridge University Press. xix, 558 p., 2002.

[11] Ronghua Li, Zhongying Chen and Wei Wu. Generalized difference methods for differential equations: Numerical analysis of finite volume methods. Pure and Applied Mathematics, Marcel Dekker. 226. New York, NY: Marcel Dekker. xv, 442 p., 2000.

[12] Peter Matus and Iryna Rybak. Difference schemes for elliptic equations with mixed derivatives. Comput. Methods Appl. Math., 4(4):494-505, 2004.

[13] I. D. Mishev. Finite volume methods on Voronoi meshes. Numerical Methods for Partial Differential Equations, 14(2):193-212, 1998.

[14] R.A. Nicolaides and D.-Q. Wang. Convergence analysis of a covolume scheme for Maxwell's equations in three dimensions. Math. Comput., 67(223):947-963, 1998.

[15] Roy A. Nicolaides and Xiaonan Wu. Covolume solutions of three-dimensional div-curl equations. SIAM J. Numer. Anal., 34(6):2195-2203, 1997.

[16] Mario Putti and Christian Cordes. Finite element approximation of the diffusion operator on tetrahedra. SIAM J. Sci. Comput., 19(4):1154-1168, 1998.

[17] Nicolas Robidoux and Steinberg Stanly. A discrete vector calculus in tensor grids. Comput. Methods Appl. Math., be published.

[18] A. A. Samarskii. Parabolic equations and difference methods for their solution. In Proc. of All-Union Conference on Differential Equations, 1958, pp. 148-160. Publishers of Armenian Ac.Sci., 1960.

[19] A. A. Samarskii. The theory of difference schemes, volume 240 of Monographs and Textbooks in Pure and Applied Mathematics. Marcel Dekker Inc., New York, 2001.

[20] A. A. Samarskii and P. N. Vabishchevich. Numerical Methods for Solving Convection-Diffusion Problems. Editorial URSS, Moscow, 2003. in Russian

[21] A.V. Solov'ev, E.V. Solov'eva, V.F. Tishkin, A.P. Favorskij and M.Yu. Shashkov. Approximation of finite-difference operators on a mesh of Dirichlet cells. Differ. Equations, 22:863-872, 1986.

[22] P.N. Vabishchevich. Finite-difference approximation of mathematical physics problems on irregular grids. Comput. Methods Appl. Math., 5(3):294-330, 2005.

[23] P.N. Vabishchevich and A.A. Samarskii. Finite difference schemes for convectiondiffusion problems on irregular meshes. Comput. Math. Math. Phys., 40(5):692704,2000 . 